\title{
A COMPARISON BETWEEN THE PERFORMANCE OF SPLIT AND INTEGRATED RF PHOTOINJECTORS*
}

\author{
J. B. Rosenzweig, S. Anderson, X. Ding, and L. Serafini ${ }^{+}$ \\ UCLA Department of Physics and Astronomy, \\ 405 Hilgard Ave., Los Angeles, CA 90095
}

\begin{abstract}
$\mathrm{RF}$ photoinjectors, the present source of choice for production of ultra-high brightness electron beams, have two basic design types: split, in which a short, high gradient rf gun is followed by a drift and a booster linac, and a lower gradient integrated photoinjector, in which the linac acceleration is connected directly to the gun. The first type is represented at UCLA by the Neptune photoinjector, the second by the newly constructed S-band PWT photoinjector. We examine, through simulation and theory, the relative merits of each type of injector, both from the point of view of the beam physics (ability of the source to produce high currents and low emittances), and of relativetechnical advantages.
\end{abstract}

\section{PHOTOINJECTOR TYPES}

The rf photoinjector is a photoelectron source embedded in a high-gradient $\mathrm{rf}$ accelerating cavity system which produces high peak current, low emittance, short pulse electron beams. These beams find application in radiation production (SASE FELs, Compton-scattering sources), as well as advanced accelerator applications (linear collider development, ultra-low emittance test beams, high current drivers for wake-field accelerators).

The photocathode "gun" region is followed by a transverse focusing element (usually a solenoid), which aids in beam size and emittance control. It additionally must be post-accelerated to bring the beam to a usable energy, and to mitigate space-charge effects. This acceleration is accomplished in a booster linac, which may be physically separated, or integrated into the same rf structure as the gun. These two configurations, termed split and integrated photoinjectors, are displayed in Figs. 1 and 2, respectively. The two devices shown are both in use at UCLA.

The first is the Neptune photoinjector[1], a device dedicated fundamental beam physics, as well as injection into advanced short-wavelength accelerator experiments, such as the plasma beatwave accelerator[2]. The Neptune photoinjector consists of a 1.6 cell high gradient 2856 $\mathrm{MHz}$ rf gun[3] derived from a family of guns developed originally at BNL, followed by $90 \mathrm{~cm}$ of drift and a postacceleration plane-wave transformer (PWT) linac[4]. Because ultra-short pulses are at a premium in this lab, a magnetic chicane[5] for pulse compression is added after the acceleration in the PWT linac from 5 to over $15 \mathrm{MeV}$. Pulse compressionof this sort, albeit at higher energies, is a common feature of ultra-short wavelength SASE FEL designs.

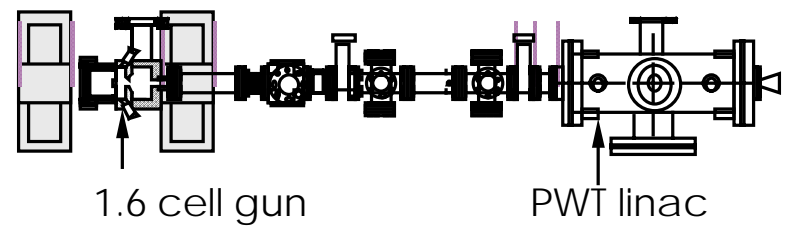

Figure 1.The UCLA Neptune photoinjector with 1.6 cell gun, focusing solenoids, $5 \mathrm{MeV}$ transport section and PWT linac.

The integrated photoinjector, which can be recognized as the original LANL design archetype, is represented at UCLA by the PWT photoinjector, a $10+2 / 2$ cell 2856 $\mathrm{MHz}$ device shown in Fig. 2. It operates at roughly the same acceleration gradient ( $60 \mathrm{MV} / \mathrm{m}$ peak) as the PWT linac in the Neptune injector, which is one-half the acceleration gradient of the 1.6 cell gun at Neptune. Thus the PWT injector can be considered a relatively low gradient device.

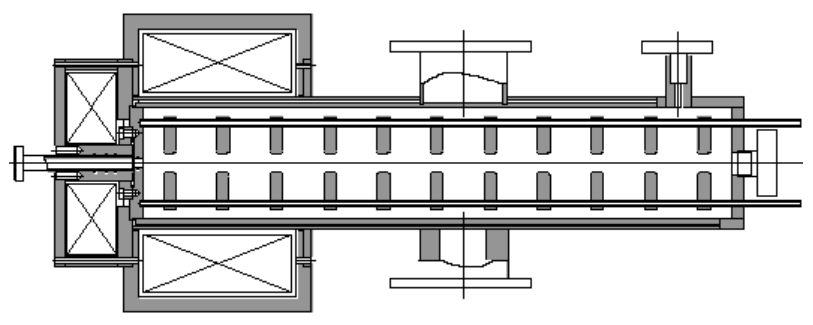

Figure 2. The UCLA/DULY PWT photoinjector, showing rf structure and compact focusing solenoid.

Both of the UCLA devices have been proposed as ultra-high brightness injector candidates for driving the LCLS x-ray FEL. While a number copies of the 1.6 cell gun have been fabricated and are now being employed, its ultimate performance is not known. Likewise, the PWT photoinjector is only now approaching commissioning, with no experimental data to verify its utility as a high brightness injector. Since we cannot rely on experimental studies to evaluate the prospects for each of these sources, we undertake here a critical compuational and theoretical comparison of the expeceted performances of the devices. 


\section{PHOTOINJECTOR BEAM PHYSICS}

The analysis and design of rf photoinjector sources for peak brightness - short pulse, high charge, and low emittance - entail a working understanding of many aspects of beam physics. We now give a cursory discussion of the physics of these beams, which are dominated by space-charge and violent acceleration effects.

The longitudinal dynamics in an rf photoinjector are characterized by violent acceleration. This is due to two effects: the need to mitigate detrimental space-charge effects, and the requirement that the beam be captured in the rf wave within the initial cell of the standing wave rf cavity. This can be quantified by defining a unitless parameter which must exceed approximately unity[6]

$$
\alpha \equiv \frac{e E_{0}}{2 k_{R F} m_{e} c^{2}}=\frac{\gamma^{\prime}}{k_{R F}}>1
$$

This parameter allows a classification of injector types: high gradient injectors have $\alpha \cong 1.5-2.5$, whereas the lowest gradient injectors operate with $\alpha \cong 1$. In split injectors, beams will typically suffer degradation in the inter-accelerator drift unless they are run at high gradient ( $\alpha=2$ at Neptune). On the other hand, the space-charge dynamics of beams in integrated photoinjectors is optimized for low gradients ( $\alpha=1$ for PWT).

The peak current at injection (leaving the cathode surface) is $I=Q / \sqrt{2} 2 \pi \sigma_{t}$. This current tends to be enhanced by the longitudinal focusing of the rf gradient at injecetion, and be diminished by the longitudinal defocusing due to space-charge. Both of these effects are asserted only close to the cathode. A one-dimensional Hamiltonian theory has been re4cently developed to analyze these effects, with the result that the pulse compression/expansion factor is expected to be

$$
\frac{d \phi_{f}}{d \phi_{0}}=\frac{2 \sin \left(\phi_{0}\right)-\phi_{0} \cdot\left[\cos \left(\phi_{0}\right)-\delta^{\prime}\right]}{1+\sin \left(\phi_{0}\right)} .
$$

Here $\phi_{0}$ is the phase at injection, and

$$
\delta^{\prime}=\frac{4 \pi r_{e} n_{b}}{e E_{0} k_{R F}}=\frac{1}{\alpha}\left[\frac{k_{p}}{k_{R F}}\right]^{2},
$$

with $n_{b}$ defined as the beam density expected with no phase compression or expansion.

Note that for low $\alpha, \phi_{0}$ is smaller, as the bunch slips more in phase while accelerating to a final optimum phase $\phi_{0}=\pi / 2$, which Eq. 2 indicates tends to compress the bunch. On the other, hand Eq. 3 predicts that the pulse lengthening due to space-charge is enhanced by running at for low $\alpha$. In order to understand how these effects compete, we must first examine the role of the plasma wave-number $k_{p}$ in photoinjectors.

Violent acceleration carries with it large transverse forces, which for an accelerator cavity terminate on a conducting (cathode) plane, gives a net first order kick to an off-axis accelerating particle. Further, this kick is rf phase dependent, and thus for a finite pulse length beam, an effective "rf" emittance is[6]

$$
\varepsilon_{R F} \propto \gamma^{\prime} k_{R F}^{2} \sigma_{r}^{2} \sigma_{z}^{2} \propto \alpha k_{R F}^{3} \sigma_{r}^{2} \sigma_{z}^{2} .
$$

The first order rf kick can be thought of as originating at the end of the structure. All other irises in the rf structure have a balance in first order inward/outward kicks, but have a second order alternating gradient focusing (of strength $\propto \gamma^{\prime 2}$ )[7]. The combined effect of the $\mathrm{rf}$ focusing can be included in envelope and matrix treatments of the beam dynamics. The analytical model has been recently verified experimentally[8].

The rf emittance is much more of a problem in the split injector than in the integrated case, because the rf kick giving rise to this effect occurs when the beam is large, at the end of the second cell, where the solenoid has not yet focused the beam. In the integrated case, the beam is generally small at the structure exit, and therefore the rf kick is diminished and the emittance contribution is negligible.

Photoinjector beams are generally space-charge dominated, meaning that the envelope dynamics are driven by space-charge forces, and not emittance "pressure". The space-charge effects give rise to plasma oscillations about an externally imposed (solenoid and rf focused) equilibria (the invariant envelope[9]). This plasma behavior in turn gives rise to emittance oscillations due to the fact that different longitudinal "slices of the beam oscillate about different equilibria. After an integer number of plasma oscillations, the beam slices are realigned in phase space, and the emittance is "compensated".

The plasma picture of the beam dynamics has allowed the development of laws for taking an optimized photoinjector design and scaling it to a different charge or rf wavelength[10]. Since we are comparing two different devices at the same rf wavelength, we only need to discuss here the charge scaling at a given rf wavelength. In this case, we simply require that the beam $n_{b}$ density is constant, and thus the beam dimensions scale as $\sigma_{i} \propto Q^{1 / 3}$. This dependence gives emittance scaling as

$$
\varepsilon_{n}=\sqrt{a Q^{4 / 3}+b Q^{8 / 3}} .
$$

where the first term is due to space-charge, with constant $a$ proportional to $k_{p}^{4 / 3} / \alpha^{3}$, and the second term is due to rf and chromatic effects. The highest brightness beams are created at lowest charge, where the emittance is mainly due to space-charge, and thus $\varepsilon_{n} \propto k_{p}^{2 / 3} / \alpha^{3 / 2}$.

All dependences of brightness can thus be understood when we assert that the plasma wave-number must scale 
as the acceleration rate, $k_{p} \propto \alpha$. For a given charge $Q$ this implies that the relative pulse lengthening is diminished for low $\alpha$. Also, at a constant $Q$, the emittance will scale as $\varepsilon_{x} \propto \alpha^{-5 / 6}$.

These predictions were tested in scaling comparisons of both optimized performance of the Neptune and PWT photoinjectors using the simulation code PARMELA. The behavior of the final rms bunch length is shown for these cases in Figs. 3 and 4, along with the "launched" rms bunch length $c T$, and the predictions of Eq. 2. The PARMELA results show excellent agreement with theory. It is seen that, while the beam launched beam is longer in the PWT (9.8 psec FWFM) than in Neptune (6 psec), that the beam compresses in the low $\alpha$ PWT, while expanding in the 1.6 cell gun, leaving the achievable current in both cases nearly identical. The expansion in the split injector is potentially troublesome (it can be enhanced by the cathode emission nonuniformities), and has been observed in initial 1.6 cell gun tests at BNL.

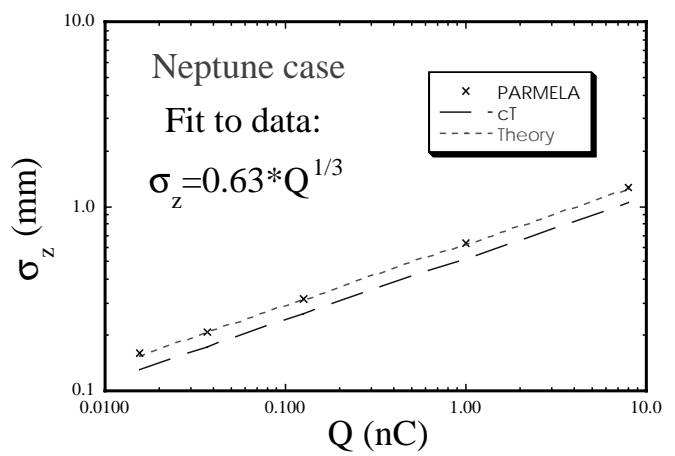

Figure 3. Neptune photoinjector bunch length vs. charge.

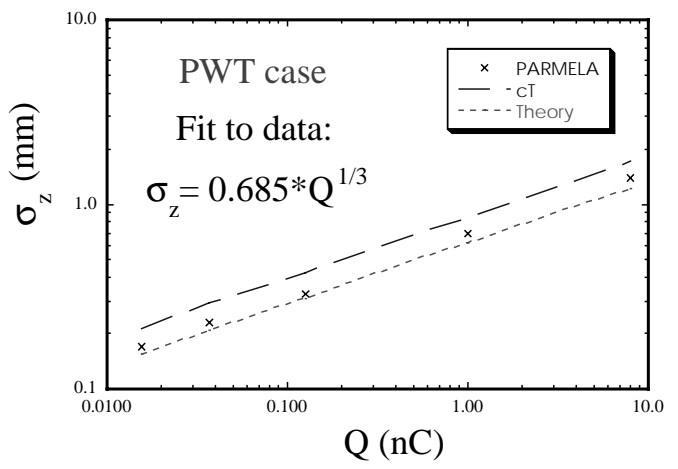

Figure 4. PWT photoinjector bunch length vs. charge.

The simulated behavior of the beam emittances as a function of charge are shown in Figs. 5 and 6 for our two cases. It can be seen that for the high $\alpha$ split photoinjector that both the contribution to the emittance due to space-charge and rf effects is smaller than for the integrated case. At one $\mathrm{nC}$, the Neptune photoinjector is expected to give normalized (rms, using $90 \%$ of the beam) $\varepsilon_{x}=0.62 \mathrm{~mm}$-mrad, while the PWT injector is expected to give $\varepsilon_{x}=1.16 \mathrm{~mm}-\mathrm{mrad}$, which is a ratio of 1.87 , which compares well with the predicted scaling ratio of 1.78. Thus we conclude that for the nominal application of creating an ultra-high brightness beam that the split photoinjector is better at $1 \mathrm{nC}$ in S-band by a factor of 3 .

These conclusions are modified by practical considerations if one allows a different choice of $\mathrm{rf}$ wavelength in the design. It has been shown that the brightness of a source scales as $k_{R F}^{2}$, which implies that one should scale the photoinjector to higher rf frequency. This can't, due to engineering constraints, be done for the split injector, and thus recent work on ultra-high brightness high $k_{R F}$ sources has focused on integrated systems.

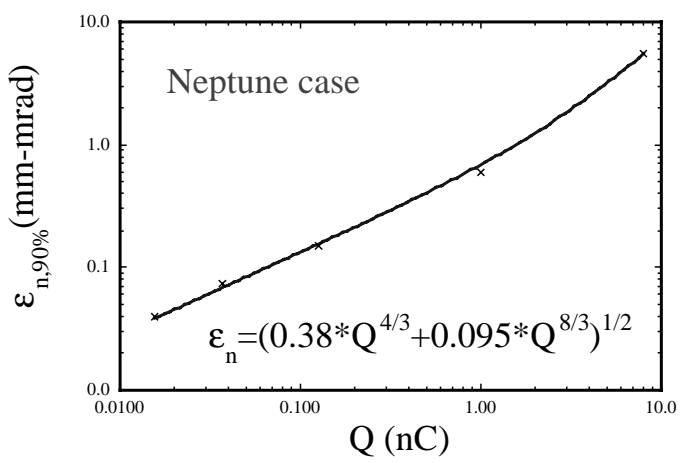

Figure 5. Neptune photoinjector emittance vs. charge.

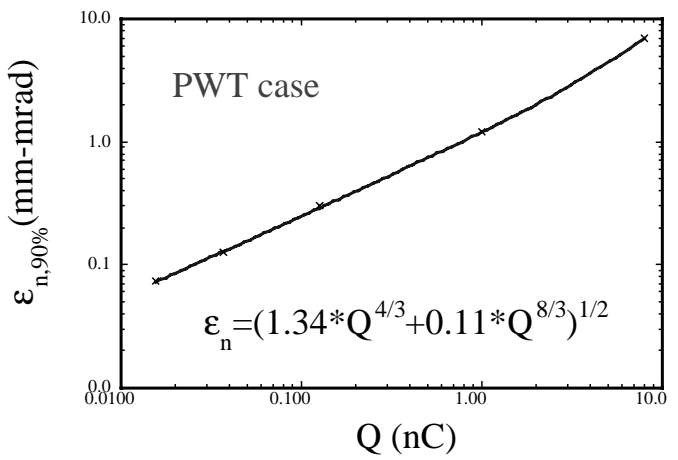

Figure 6. PWT photoinjector emittance vs. charge.

"Work supported by US DoE DoE Contracts DE-FG03-92ER40693 and DE-FG03-98ER45693.

\# Email: rosenzweig@physics.ucla.edu

${ }^{+}$INFN-Milano, Univ. Milan..

\section{REFERENCES}

1. J. Rosenzweig, et al., NIM A 410, 437 (1998).

2. C. Clayton, et al., ., NIM A 410, 437 (1998).

3. D.T.Palmer, et al., Proc. PAC'97, 2687 (IEEE,1998)

4. R. Zhang, et al., Proc. of the 1995 PAC (IEEE,1996)

5. J.B. Rosenzweig, N. Barov and E. Colby, IEEE Trans. Plasma Sci. 24, 409 (1996).

6. K.J. Kim, NIM A 275, 201 (1989).

7. J. Rosenzweig and L. Serafini, Phys. Rev. E 49, 1499 (1994).

8. S. Reiche, et al., Phys, Rev. E 56, 3572 (1997).

9. Luca Serafini and James Rosenzweig, Phys. Rev. E 55, 7565 (1997).

10. J. Rosenzweig, and E. Colby, Proc. 1995 Advanced Accelerator Concepts Workshop 337 (AIP, 1996). 